\title{
SPECTROSCOPY FROM THE HUBBLE SPACE TELESCOPE COSMIC ORIGINS SPECTROGRAPH OF THE SOUTHERN NOVA-LIKE BB DORADUS IN AN INTERMEDIATE STATE
}

\author{
Patrick Godon ${ }^{1,8}$, Edward M. Sion ${ }^{1}$, Boris T. Gänsicke ${ }^{2}$, Ivan Hubeny ${ }^{3}$, Domitilla de Martino ${ }^{4}$, Anna F. Pala ${ }^{2}$, \\ Pablo Rodríguez-GiL ${ }^{5,6}$, Paula Szkody ${ }^{7}$, and Odette Toloza ${ }^{2}$ \\ ${ }^{1}$ Department of Astrophysics \& Planetary Science, Villanova University, Villanova, PA 19085, USA; patrick.godon@villanova.edu, edward.sion@ villanova.edu \\ ${ }^{2}$ Department of Physics, University of Warwick, Coventry CV4 7AL, UK; A.F.Pala@warwick.ac.uk, O.F.C.Toloza@warwick.ac.uk, \\ boris.gaensicke@warwick.ac.uk \\ ${ }_{4}^{3}$ Steward Observatory, The University of Arizona, Tucson, AZ 85721, USA; hubeny@as.arizona.edu \\ ${ }^{4}$ INAF aLS Osservatorio Astronomico di Capodimonte, Napoli, I-80131, Italy; demartino@na.astro.it \\ ${ }^{5}$ Instituto de Astrofísica de Canarias, Vía Láctea, s/n, La Laguna, E-38205 Santa Cruz de Tenerife, Spain; prguez@iac.es \\ ${ }^{6}$ Departmento de Astrofísica, Universidad de La Laguna, La Laguna, E-38206 Tenerife, Spain \\ ${ }^{7}$ Department of Astronomy, University of Washington, Seattle, WA 98195, USA; szkody@astro.washington.edu \\ Received 2016 August 4; revised 2016 October 10; accepted 2016 October 10; published 2016 December 13
}

\section{ABSTRACT}

We present a spectral analysis of the spectrum from the Hubble Space Telescope Cosmic Origins Spectrograph $(H S T / \mathrm{COS})$ of the southern VY Scl nova-like variable BB Doradus, obtained as part of a Cycle $20 \mathrm{HST} / \mathrm{COS}$ survey of accreting white dwarfs (WDs) in cataclysmic variables. BB Dor was observed with COS during an intermediate state with a low mass accretion rate, thereby allowing an estimate of the WD temperature. The results of our spectral analysis show that the WD is a significant far-ultraviolet (FUV) component of the spectrum with a temperature of about $35,000-50,000 \mathrm{~K}$, assuming a WD mass of $0.80 M_{\odot}(\log (g)=8.4)$. The disk, with a mass accretion rate of $\approx 10^{-10} M_{\odot} \mathrm{yr}^{-1}$, contributes about $1 / 5$ to $1 / 2$ of the FUV flux.

Key words: accretion, accretion disks - binaries: close - novae, cataclysmic variables - stars: individual (BB Doradus) - ultraviolet: stars - white dwarfs

\section{INTRODUCTION}

\subsection{Accretion Rates and WD Temperatures in CVs}

Cataclysmic variables (CVs) are close binaries in which a white dwarf (WD) accretes matter from a low-mass companion donor star by means of either an accretion disk around the WD or an accretion column, the latter when the WD has a strong magnetic field (Warner 1995). When enough material has been accreted onto the surface of the WD, about every $10^{3}-10^{5} \mathrm{yr}$ or so, the hydrogen-rich envelope undergoes a thermonuclear runaway - the classical nova explosion (Starrfield 1971).

In the standard model (Rappaport et al. 1982, 1983), CVs are believed to evolve from long to short binary orbital period $(P)$ driven by angular momentum losses (AMLs). Magnetic stellar wind braking is the dominant AML mechanism at long periods, and it shuts down around $P \sim 3 \mathrm{hr}$, as the secondary becomes fully convective. Since the mass-loss rate is suddenly reduced, the secondary contracts and the system becomes a detached binary, continuing to evolve to shorter periods $(P<3 \mathrm{hr})$, driven now by gravitational wave emission as the dominant AML mechanism. Around $P \sim 2 \mathrm{hr}$, the mass transfer restarts as the Roche lobe makes contact again with the donor. The system then reappears as active and continues to evolve, reducing its period. The majority of CVs in our galaxy should have already evolved to a minimum period near 80 minutes and now have degenerate brown dwarf-like secondaries (Howell et al. 2001; Goliasch \& Nelson 2016). However, some discrepancies have arisen between the observations and the standard model, prompting suggestions of additional (or alternative) theoretical scenarios (see, e.g., Knigge et al. 2011 and references therein for a review of the problem).

\footnotetext{
${ }^{8}$ Visiting at the Henry A. Rowland Department of Physics and Astronomy, The Johns Hopkins University, Baltimore, MD 21218, USA.
}

In order to differentiate between the different possible theoretical paths, one needs to know the distribution of the time-averaged mass accretion rate $(\langle\dot{M}\rangle)$ of $\mathrm{CVs}$ as a function of the binary orbital period $P$ (Townsley \& Bildsten 2005). Alternatively, the distribution of the WD effective surface temperature $T_{\mathrm{wd}}$ versus $P$ can also be used (Townsley \& Gänsicke 2009). Therefore, assessing the mass accretion and/ or WD effective temperature of CV systems has become a primary goal.

The mass accretion rate can be obtained by fitting theoretical accretion disk spectra to far-ultraviolet (FUV) spectra of CV systems accreting a high rate, and the WD temperature is obtained by fitting synthetic photospheric spectra to FUV spectra of CV systems in which the accretion is greatly reduced.

Nova-like variables (NLs) form a class of nonmagnetic or weakly magnetic $\mathrm{CVs}$ characterized by their high mass accretion rate, in which the accretion disk is the dominant source of ultraviolet and optical light (la Dous 1990, 1991). These systems are often observed for the study of the accretion disk and to determine the mass accretion rate $\dot{M}$, since the WD cannot be detected because it is outshone by the brightness of the accretion structures. This prevents determination of the WD parameters. The VY Scl subclass of NLs consists of systems that occasionally drop into a low-brightness state (Hack \& la Dous 1993), thereby allowing the study of the WD and its companion. Because of that, the VY Scl subclass of NLs are of special interest, as they provide the opportunity to assess the effective surface temperature of their WDs. So far, only a handful of NL systems have been studied in the low state, revealing hot WDs with a temperature $\sim 30,000$ to $\sim 50,000 \mathrm{~K}$ (Townsley \& Gänsicke 2009; Rodríguez-Gil et al. 2015). BB Doradus is a member of this subclass of NL systems and is therefore of special interest. 


\subsection{The VY Scl Nova-like CV BB Doradus}

BB Doradus, also known as EC 05287-5847, was spectroscopically identified as a CV from the Edinburgh-Cape Survey (Chen et al. 2001). It was not brighter than $m_{v} \sim 16.5$ until 1992 November, after which it went into a bright state, reaching $M_{\mathrm{v}} \sim 14.6-13.6$. Since then, it has been observed to exhibit occasional low states (Rodríguez-Gil et al. 2012; Schmidtobreick et al. 2012). A photometric coverage of 45 days by Patterson (2002) revealed superhumps and a photometric modulation of 0.14923 day (or $3.58 \mathrm{hr}$ ). More recently, however, a radial velocity study, using for the first time the $\mathrm{H} \alpha$ radial velocities during the low state (Rodríguez-Gil et al. 2012), put the orbital period at $0.154095 \pm 0.000003$ day $(3.69828 \pm 0.00007 \mathrm{hr})$, as expected for VY Scl stars, which usually have orbital periods between 3 and $4 \mathrm{hr}$. Rodríguez-Gil et al. (2012) suggest that the modulation of 0.14923 day derived by Patterson (2002) may be identified as a negative superhump. BB Dor also exhibits quasi-periodic oscillations (QPOs) with timescales between 878 and $1154 \mathrm{~s}$ (Chen et al. 2001). Because of its very narrow Balmer lines (with wings extending only $\sim 650 \mathrm{~km} \mathrm{~s}^{-1}$ from the line center), BB Dor is suspected to be a low-inclination system (Chen et al. 2001). The Galactic reddening value in the direction of BB Dor, as retrieved from the online GALEX MAST archive, is very small, $E(B-V)=0.035$; therefore we assume that the reddening of $\mathrm{BB}$ Dor is negligible, $E(B-V) \approx 0$.

We observed BB Dor on 2007 July 9 with the Far Ultraviolet Spectroscopic Explorer (FUSE), just days before the fatal failure of the reaction wheel of the telescope (Godon et al. 2008), and found it in a high state with a visual magnitude $\sim 13.6-14.6$, revealing a mass accretion rate of $\sim 10^{-9} M_{\odot} \mathrm{yr}^{-1}$ (for a standard WD with $\log (g)=8.4$ ), and a distance estimate of $\sim 665 \mathrm{pc}$ (Godon et al. 2008). Taking into account the contribution of a hot $\left(T_{\text {eff }}=32,000 \mathrm{~K}\right) \mathrm{WD}$ in addition to the disk pushed the distance to $\sim 700 \mathrm{pc}$, but overall, the inclusion of the WD did not significantly improve the fit because the hot WD contributed only $10 \%$ of the flux.

More recently, Pala et al. (2016) observed BB Dor with the Hubble Space Telescope Cosmic Origins Spectrograph (HST/ $\mathrm{COS}$ ), as part of a 122 orbit $H S T / \mathrm{COS}$ program to measure the WD effective temperature in CVs in the low state or quiescence. In that analysis, Pala et al. modeled the COS spectra of CVs using synthetic spectra of the WD plus a second component in the form of a simple power law or blackbody but they did not include the contribution from an accretion disk. All the systems were observed when the disk contribution to the FUV flux was expected to be negligible. BB Dor, however, was observed in an intermediate state (see below), and the broad hydrogen Ly $\alpha$ absorption profile of the WD was not detected in its COS spectrum; for that reason it was not modeled by Pala et al. (2016).

In the present work, we extend the spectral analysis of the COS spectrum of BB Dor to also include the disk component; that is, our spectral modeling includes synthetic spectra of a WD with an accretion disk. We also take into account the results of the FUSE spectroscopic analysis (Godon et al. 2008). By doing so, we aim to obtain constraints on both the WD effective surface temperature and the mass accretion rate of $\mathrm{BB}$ Dor during its intermediate state.

\section{THE COS SPECTRUM OF BB DOR}

Data from the American Association of Variable Star Observers showed that BB Dor dropped from its usual high state $\left(m_{\mathrm{v}} \approx 15\right)$ into a low state in 2013 mid-January, reaching a magnitude of $\sim 19-19.5$, thereby triggering the $H S T / \mathrm{COS}$ observations of it. However, the luminosity of the system started to increase on January 19, and as a consequence, at the time of the COS observations (2013 February 13, 25 days after its deep minimum), BB Dor was found in an intermediate state, with a magnitude of 17.5-18.0. It took another $\sim 25$ days to return to its high state.

The COS instrument was set with the detector in the FUV channel configuration, with the G140L grating centered at a wavelength of $(C E N W A V E=) 1105 \AA$, covering the wavelength range $1110-2150 \AA$, with a resolution of $R \approx 3000$. The photons were collected through the Primary Science Aperture with a diameter of $2.5 \mathrm{arcsec}$. The COS data consist of a total of $7272 \mathrm{~s}$ of good exposure in TIME-TAG mode. We used the four FP-POS positions to improve the signal-to-noise ratio and minimize the effects of flat-field artifacts. The data (LC1VVD010) consisted of five subexposures, because one of the FP-POS positions spread over two consecutive spacecraft orbits. A final spectrum was then constructed by coadding the five X1D files generated by CALCOS (v3.1; Hodge et al. 2007; Hodge 2011).

The longer wavelengths beyond $2000 \AA$ were found to be very noisy and that portion of the spectrum had to be discarded. Consequently, the final spectrum starts at $1117 \AA$ and ends at $2000 \mathrm{~A}$. The spectrum is presented in Figure 1.

Strong emission lines from low and high ionization species have been identified and are annotated in Figure 1. The wavelength region 1180-1250 $\mathrm{A}$ does not show any discernible hydrogen Ly $\alpha$ profile/wings (from the WD and/or accretion disk) due to the many emission features in that region. In fact, the spectrum is contaminated with strong and broad geocoronal emission of Ly $\alpha(1216 \AA)$ and O I (1302 $\AA$ ). At the longer wavelengths, $\sim 1850-1900 \AA$, we identify emission lines from Al III (1857 and $1862 \AA$ ), and there seem to be additional emission features beyond $1900 \AA$ that remain unidentified. Because of this, we ignore the portion of the spectrum in the longer wavelengths $(\lambda>1850 \AA)$ in our modeling (our spectral modeling does not include emission lines).

Of all the emission lines from the source, the C IV line is the strongest and best resolved. We therefore use it to assess the inclination of the system because this line is believed to be forming in the hottest region in the disk, namely, the very inner disk $r \sim 1-2 R_{\mathrm{wd}}$. The C IV (1u) line is made of a doublet with rest wavelengths at 1548.20 and $1550.78 \AA$. The modeling of the doublet with a double Gaussian gives an FWHM of $6.85 \AA$ (for each Gaussian), and twice as much for the maximum broadening at their base. This translates into a velocity of $\sim 650 \mathrm{~km} \mathrm{~s}^{-1}$ for the FWHM and $\sim 1300 \mathrm{~km} \mathrm{~s}^{-1}$ for the maximum broadening. Assuming that the maximum broadening is due to the largest Keplerian speed in the disk, obtained at $r \sim R_{\mathrm{wd}}$, one obtains an inclination of $\sim 20^{\circ}$ for the disk/ binary for an average CV WD mass of $0.8 M_{\odot}$ with a maximum Keplerian speed of $\sim 3900 \mathrm{~km} \mathrm{~s}^{-1}$. Assuming a larger radius for the region where the CIV line forms, $r \approx 2 R_{\mathrm{wd}}$, gives an inclination of $\sim 30^{\circ}$.

There are also some absorption lines, mostly at short wavelengths. All the absorption lines are much narrower than the emission lines. We present the lines in Figure 2. We 


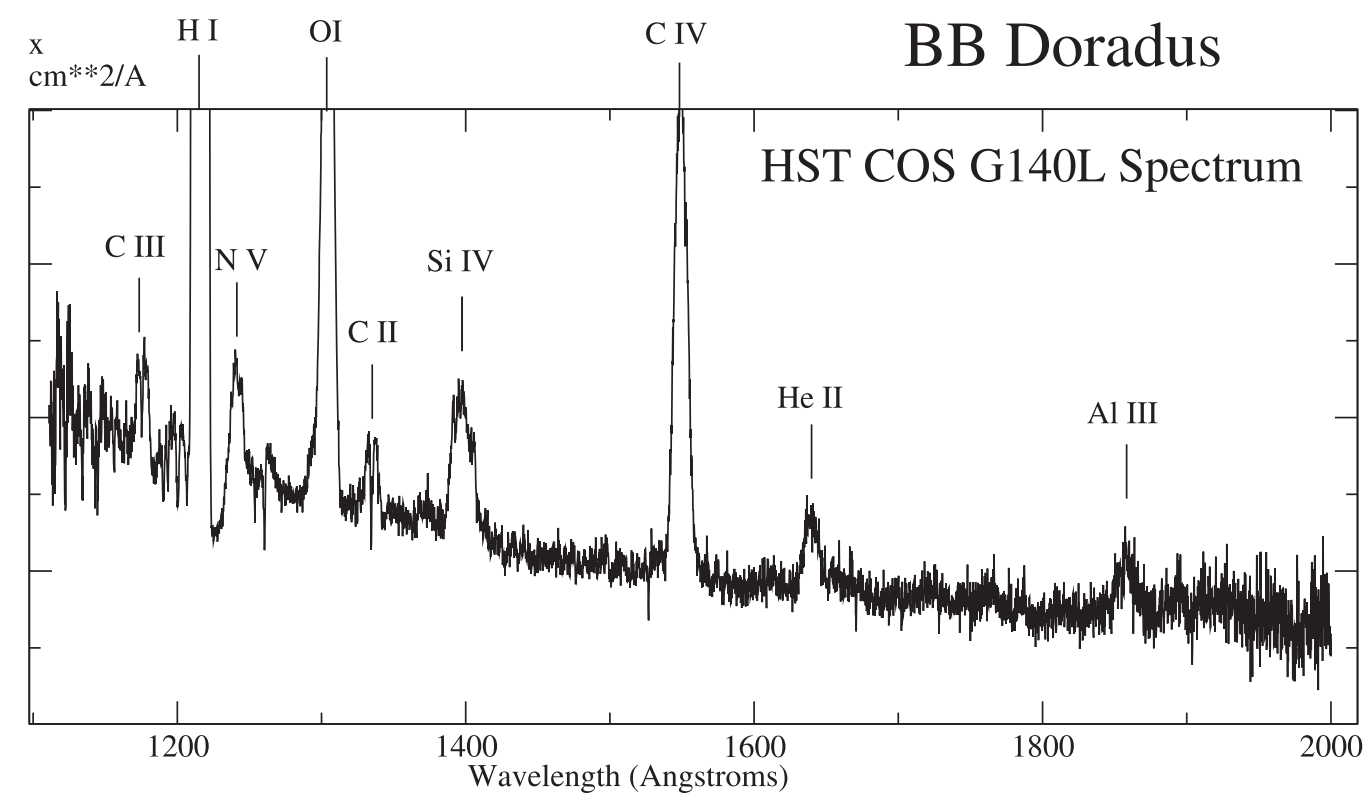

Figure 1. $H S T / \mathrm{COS}$ spectrum of BB Dor is shown with line identification. The spectrum presents some broad and strong emission lines of high ionization species

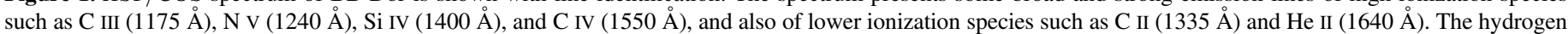
Ly $\alpha(1216 \AA)$ and O I (1302 $⿱$ ) features are due to geocoronal contamination. Toward the longer wavelengths, after $1700 \AA$ and especially after $1900 \AA$, there are additional weak but broad emission features that remain unidentified. The region $\lambda>2000 \AA$ is dominated by noise and has been discarded for clarity. For that reason in the modeling we limit the fitting to $\lambda<1850 \AA$.

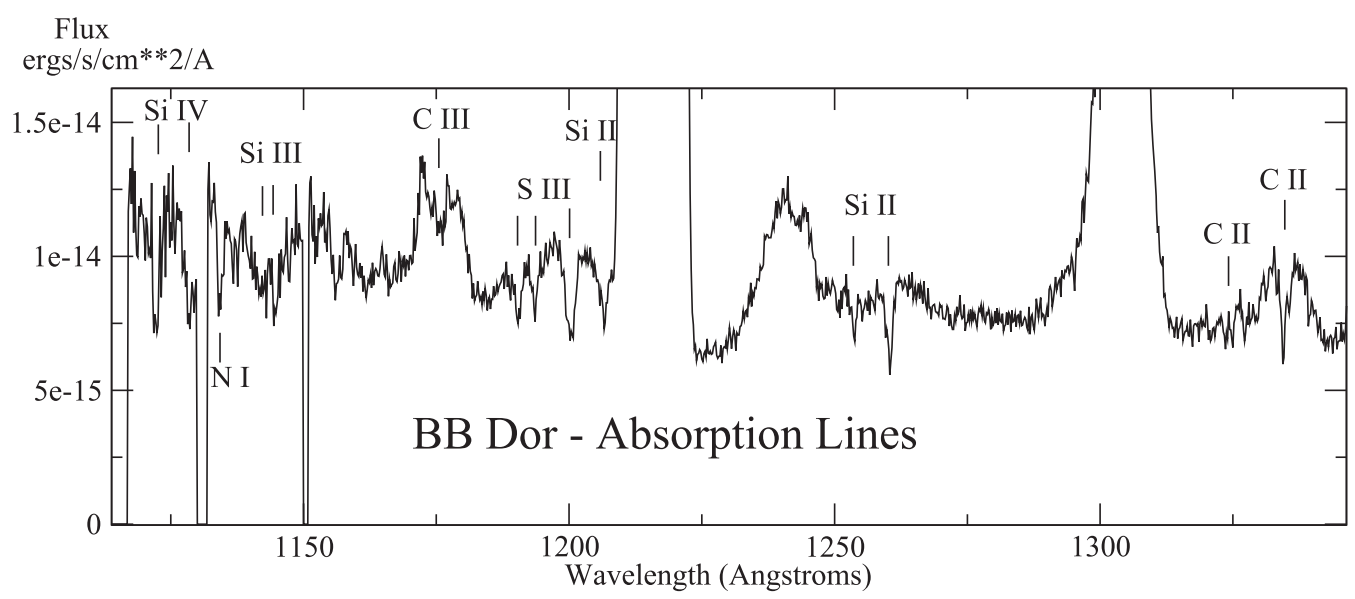

Figure 2. Absorption lines are tentatively identified and annotated in the shorter wavelength region of the COS spectrum of BB Dor. The nitrogen line N I (1134 $\AA$ ) is possibly geocoronal. The two narrow gaps $(\sim 1130$ and $\sim 1150 \AA)$ are due to further post-processing of the spectrum, namely, cutting off unreliable data in the subexposures to remove small detector artifacts that could be misidentified as absorption lines.

tentatively identify the following lines: Si IV (1122.5 and $1128.3 \AA)$, Si III (1140.6, 1142, and $\sim 1145 \AA$ ), C III $(\sim 1175$ $\AA), S$ III (1190.2, 1194.2, and $1201 \AA)$, Si III (1206.5 ̊), Si II $(1260 \AA), C$ II $(1334.5$ and $1335.7 \AA)$, Si III $(\sim 1500 \AA)$, and Si II (1526 $⿱$ A). The nitrogen line N I (1134 $\AA$ ) is most probably terrestrial in origin and has often been observed in FUSE spectra of CVs. Since the absorption lines might potentially originate in the WD photosphere or the accretion disk (when seen at low inclination), we further discuss them in Sections 4 and 5 .

A comparison of the continuum flux level of the COS spectrum to that of the archival FUSE spectrum (taken on 2007 July 9) shows that the COS continuum flux level is lower than the FUSE continuum flux level by a factor of 20. Namely, in the overlap region $\sim 1110-1185 \AA$ the FUSE continuum reaches $\sim 2 \times 10^{-13} \mathrm{erg} \mathrm{s}^{-1} \mathrm{~cm}^{-2} \AA^{-1}$, while the COS continuum reaches $\sim 1 \times 10^{-14} \mathrm{erg} \mathrm{s}^{-1} \mathrm{~cm}^{-2} \AA^{-1}$. Though the system was not caught in a deep low state, this fortunate occurrence gives us the unique opportunity to study possible photospheric emission from the $\mathrm{WD}$, because the mass accretion rate must have dropped substantially, thereby possibly exposing the WD.

In order to assess the contribution of the WD to the total flux, we use the TIME-TAG data to generate three light curves. A first light curve is generated by integrating all the data over the entire wavelength coverage except for the known airglow emission lines of Ly $\alpha, \mathrm{O}$ I, and N I; a second light curve is made with the C IV line (1535-1564 $⿱$ ) ; and a third light curve is made using the continuum in the range 1424-1523 $\AA$. In Figure 3 we show the three light curves, with each normalized to one. The continuum varies in the same way as the C IV emission, which we know does not come from the WD. Therefore, if the continuum is the sum of the disk and WD, the 


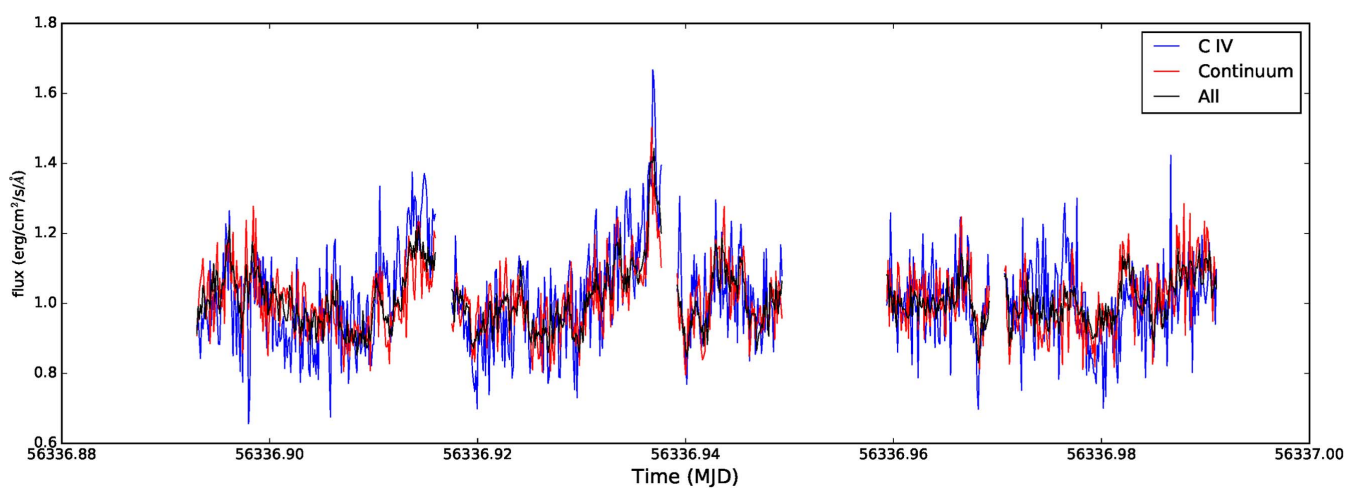

Figure 3. Light curves are shown for (i) the entire spectral range of COS (in black), (ii) the carbon line only (1424-1523 $\AA$; in blue), and (iii) the continuum in the range $1535-1564 \AA$ (in red). The five segments correspond to the five exposures. The flux has been normalized to one and the time is given using the modified Julian date.

Table 1

Results of Synthetic Spectral Modeling

\begin{tabular}{|c|c|c|c|c|c|c|}
\hline $\begin{array}{l}\text { Model } \\
\text { Number }\end{array}$ & $\begin{array}{c}T_{\mathrm{wd}} \\
\left(10^{3} \mathrm{~K}\right)\end{array}$ & $\begin{array}{c}\log (\dot{M}) \\
\left(M_{\odot} \mathrm{yr}^{-1}\right)\end{array}$ & WD/Disk & $\begin{array}{c}d \\
(\mathrm{pc})\end{array}$ & $\chi_{\nu}^{2}$ & Figure \\
\hline 1 & 23.0 & $\ldots$ & WD & 291 & 3.07 & $\cdots$ \\
\hline 2 & 27.0 & $\ldots$ & WD & 412 & 1.98 & $\ldots$ \\
\hline 3 & 30.0 & $\ldots$ & WD & 515 & 1.92 & 4,5 \\
\hline 4 & 35.0 & $\ldots$ & WD & 692 & 1.94 & 6 \\
\hline 5 & 40.0 & $\ldots$ & WD & 864 & 1.98 & $\ldots$ \\
\hline 6 & 50.0 & $\ldots$ & WD & 1052 & 2.17 & $\cdots$ \\
\hline 7 & $\ldots$ & -8.0 & disk & 6808 & 1.36 & $\cdots$ \\
\hline 8 & $\ldots$ & -8.5 & disk & 4288 & 1.32 & 7 \\
\hline 9 & $\cdots$ & -9.0 & disk & 2545 & 1.36 & $\cdots$ \\
\hline 10 & $\ldots$ & -9.5 & disk & 1356 & 1.87 & 8 \\
\hline 11 & $\ldots$ & -10.0 & disk & 679 & 4.66 & $\ldots$ \\
\hline 12 & $\ldots$ & -10.5 & disk & 322 & 12.14 & $\ldots$ \\
\hline 13 & 30.0 & -8.0 & $0.6 / 99.4$ & 6828 & 1.36 & $\cdots$ \\
\hline 14 & 50.0 & -8.0 & $2.4 / 97.6$ & 6890 & 1.37 & $\cdots$ \\
\hline 15 & 30.0 & -8.5 & $1.5 / 98.5$ & 4319 & 1.32 & $\ldots$ \\
\hline 16 & 50.0 & -8.5 & $6 / 94$ & 4415 & 1.33 & $\ldots$ \\
\hline 17 & 30.0 & -9.0 & $4 / 96$ & 2598 & 1.35 & $\ldots$ \\
\hline 18 & 50.0 & -9.0 & $15 / 85$ & 2754 & 1.34 & $\ldots$ \\
\hline 19 & 25.0 & -9.5 & $6 / 94$ & 1400 & 1.85 & $\ldots$ \\
\hline 20 & 35.0 & -9.5 & $21 / 79$ & 1524 & 1.49 & 9 \\
\hline 21 & 48.0 & -9.5 & $37 / 63$ & 1693 & 1.31 & $\cdots$ \\
\hline 22 & 60.0 & -9.5 & $46 / 54$ & 1817 & 1.31 & $\cdots$ \\
\hline 23 & 35.0 & -10.0 & $53 / 47$ & 967 & 1.47 & $\cdots$ \\
\hline 24 & 40.0 & -10.0 & $65 / 35$ & 1094 & 1.21 & 10 \\
\hline 25 & 45.0 & -10.0 & $70 / 30$ & 1175 & 1.19 & $\ldots$ \\
\hline 26 & 50.0 & -10.0 & $74 / 26$ & 1244 & 1.23 & $\ldots$ \\
\hline 27 & 27.0 & -10.5 & $66 / 34$ & 516 & 2.79 & $\ldots$ \\
\hline 28 & 30.0 & -10.5 & $76 / 24$ & 602 & 1.68 & $\ldots$ \\
\hline 29 & 35.0 & -10.5 & $86 / 14$ & 761 & 1.23 & 11 \\
\hline 30 & 40.0 & -10.5 & $91 / 9$ & 920 & 1.32 & $\ldots$ \\
\hline
\end{tabular}

fact that the variability ( $\sim 20 \%$ of the total flux) is produced by the disk indicates that the WD cannot contribute more than $\sim 80 \%$ of the flux.

In our analysis we model the COS spectrum of BB Dor with WD models, disk models, and composite models that include the contribution from both the WD photosphere and an accretion disk. Our main goal is to derive the WD effective surface temperature and the mass accretion rate of the system, to add one data point to the handful of NL systems with known $T_{\text {eff }}$. Our spectral analysis technique is described in the next section.

\section{SYNTHETIC SPECTRAL MODELING AND ANALYSIS}

In order to derive the mass accretion rate $\dot{M}$ and the WD temperature $T_{\text {eff }}$, we carry out a quantitative comparison (a "fit") of the observed spectrum to theoretical disk and WD spectra for a wide range of parameters.

The synthetic model spectra of the WD stellar atmosphere are generated using the FORTRAN suite of codes TLUSTY, SYNSPEC, and ROTIN (Hubeny 1988; Hubeny \& Lanz 1995). The basic input parameters are the stellar effective surface temperature, surface gravity, and chemical composition. In a first step, a converging stellar atmospheric structure is computed by successive runs of TLUSTY. The TLUSTY model atmospheres have hydrogen and helium taken explicitly, i.e., their selected levels are treated in non-LTE, and the bound-bound and bound-free transitions between these levels determine the total opacity and emissivity. Carbon, nitrogen, and oxygen are treated implicitly, i.e., they contribute only to the particle and charge conservation, assuming LTE. Next, a run of the spectral synthesis code SYNSPEC, using the output structure of the model atmosphere from TLUSTY as input is done. In this step, all opacity sources, i.e., the lines of all other elements, are considered (unless it is specifically required not to). The short code, ROTIN, is then used to account for the projected stellar rotational velocity (and/or instrumental) broadening, including the effect of limb darkening.

In this manner, we generate WD stellar photospheric models with effective temperatures ranging from $\sim 15,000$ to $\sim 60,000 \mathrm{~K}$ in increments of $500-1000 \mathrm{~K}$. The NLTE treatment is turned on for the hot WD models $\left(T_{\text {eff }}>35,000 \mathrm{~K}\right)$, using the approximate NLTE treatment of lines option in SYNSPEC (Hubeny \& Lanz 1995). We chose here a value of $\log (g)=8.4$, which matches a WD mass of $0.80 M_{\odot}$, a rounded value of the average WD mass in CVs of $0.83 \pm 0.23 M_{\odot}$ (Zorotovic et al. 2011). Since the spectrum has emission lines and thus a disk component, we use only solar composition (set up in SYNSPEC) because it would be impossible to derive chemical abundances for such a spectrum. We vary the stellar rotational velocity $V_{\text {rot }} \sin (i)$ from 100 to $500 \mathrm{~km} \mathrm{~s}^{-1}$ in steps of $50 \mathrm{~km} \mathrm{~s}^{-1}$. The WD rotation rate $\left(V_{\text {rot }} \sin (i)\right)$ is determined by fitting the WD model to the spectrum while paying careful attention to the line profiles. The mass-radius relation from Wood (1990), for carbon-oxygen and non-zero-temperature WDs, is used to obtain the radius of the WD. 

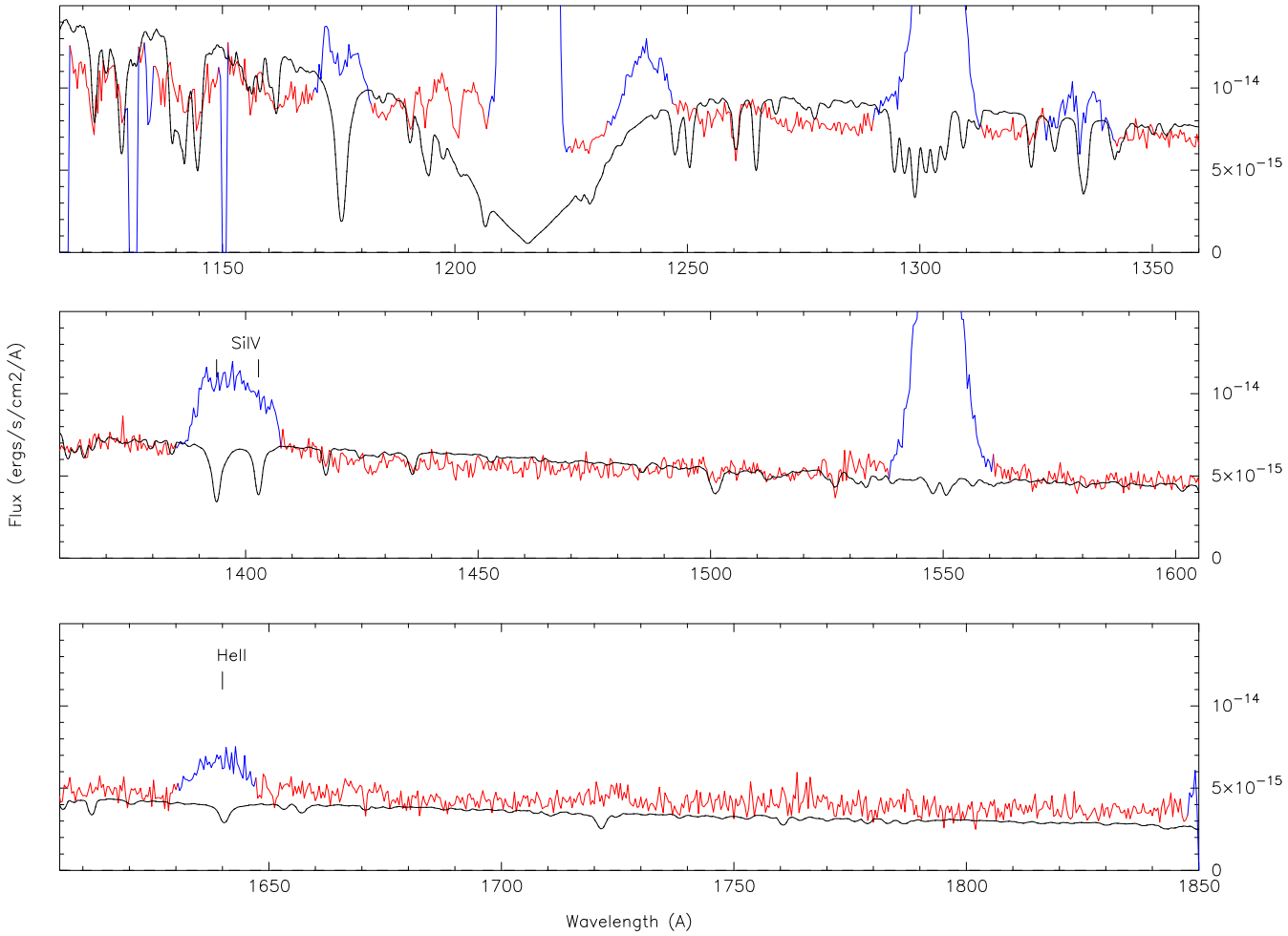

Figure 4. WD model fit (in black) to the COS spectrum of BB Dor (in red). The blue portions indicate the regions of the spectrum that have been masked due to the strong emission features. The WD has a temperature $T=30,000 \mathrm{~K}$, gravity $\log (g)=8.4$, solar composition, and projected rotational velocity of $200 \mathrm{~km} \mathrm{~s}{ }^{-1}$. This is the best-fit (least $\chi^{2}$ ) WD model. Its slope is slightly too steep and its hydrogen Ly $\alpha$ profile does not fit the observed spectrum. It is likely that the Ly $\alpha$ region $(1170-1250 \AA$ A $)$ is entirely dominated by broad emission lines.

The accretion disk spectra were generated assuming that the disk is made of a collection of annuli, where each annulus has a temperature $T(r)$ and gravity $\log (g(r))$ given by the standard disk model (Shakura \& Sunyaev 1973; Pringle 1981), for a given central mass $M_{\mathrm{wd}}$ and accretion rate $\dot{M}$. For each annulus, an atmosphere model was generated using TLUSTY, followed by a run of SYNSPEC to obtain a spectrum of the annulus. The contributions of all the annuli were combined using the code DISKSYN, and a final disk spectrum was obtained for any given inclination angle (we assume the disk to lie in the orbital plane of the binary). In the present work we use the public grid of disk models from Wade \& Hubeny (1998), in which a detailed explanation of the entire numerical procedure is given.

In our analysis, we use a grid of models of synthetic spectra of WDs and accretion disks covering a wide range of values of the WD temperature $T_{\text {eff }}$, gravity $\log (g)$, projected rotational velocity $V_{\text {rot }} \sin (i)$, inclination $i$, mass accretion rate $\dot{M}$, and abundances. The grid of disk models has the following inclination angles: $i=18^{\circ}, 41^{\circ}, 60^{\circ}, 75^{\circ}$, and $81^{\circ}$. Since the inclination of the system is low (Chen et al. 2001; Schmidtobreick et al. 2012), and based on our assessment in the previous section, we use $i=18^{\circ}$, the lowest value in the grid of models. Furthermore, as the flux level in the COS spectrum is 20 times lower than in the FUSE spectrum, we expect our COS spectral analysis to reveal a mass accretion rate lower than that derived from the FUSE spectrum, namely $\dot{M}_{\mathrm{COS}}<10^{-9} M_{\odot} \mathrm{yr}^{-1}$.

The distance to the system is not known accurately, but the results of the FUSE analysis implied a distance $d<1 \mathrm{kpc}$ (Godon et al. 2008). Rodríguez-Gil et al. (2012) estimated a distance of $d \sim 1-2 \mathrm{kpc}$ based on a WD temperature of $30,000 \mathrm{~K}$ and a secondary spectral type of M3-M4. The spectrum exhibits few absorption lines, and, as stated earlier, we decided to use solar abundances in our modeling. With our assumption of a standard CV WD mass of $0.8 M_{\odot}$, we keep $\log (g)$ constant at 8.4 . Therefore, the parameters that we vary in our model fits are the temperature of the WD, its rotation rate, and the mass accretion rate of the disk.

Before carrying out a fit of the spectrum we mask portions of the spectrum with emission lines, since our synthetic spectral code does not generate emission lines.

After having generated grids of models for the COS spectrum of BB Dor, we use FIT (Press et al. 1992), a $\chi^{2}$ minimization routine, to compute the reduced $\chi_{\nu}^{2}\left(\chi^{2}\right.$ per number of degrees of freedom $\nu$ ) and scale factor (which gives the distance) for each model fit. While we use a $\chi^{2}$ minimization technique, we do not blindly select the models with least $\chi^{2}$, but we examine the models that best fit some of the spectral features and that are most consistent with the values of the system parameters.

\section{RESULTS}

\subsection{Single WD Modeling}

Despite the facts that the hydrogen Ly $\alpha$ absorption feature is not detected in the spectrum (Pala et al. 2016), and that the WD cannot contribute more than $\sim 80 \%$ of the flux, we decide to first model the spectrum with a single WD alone. Such a modeling will confirm that a WD alone is not enough to 

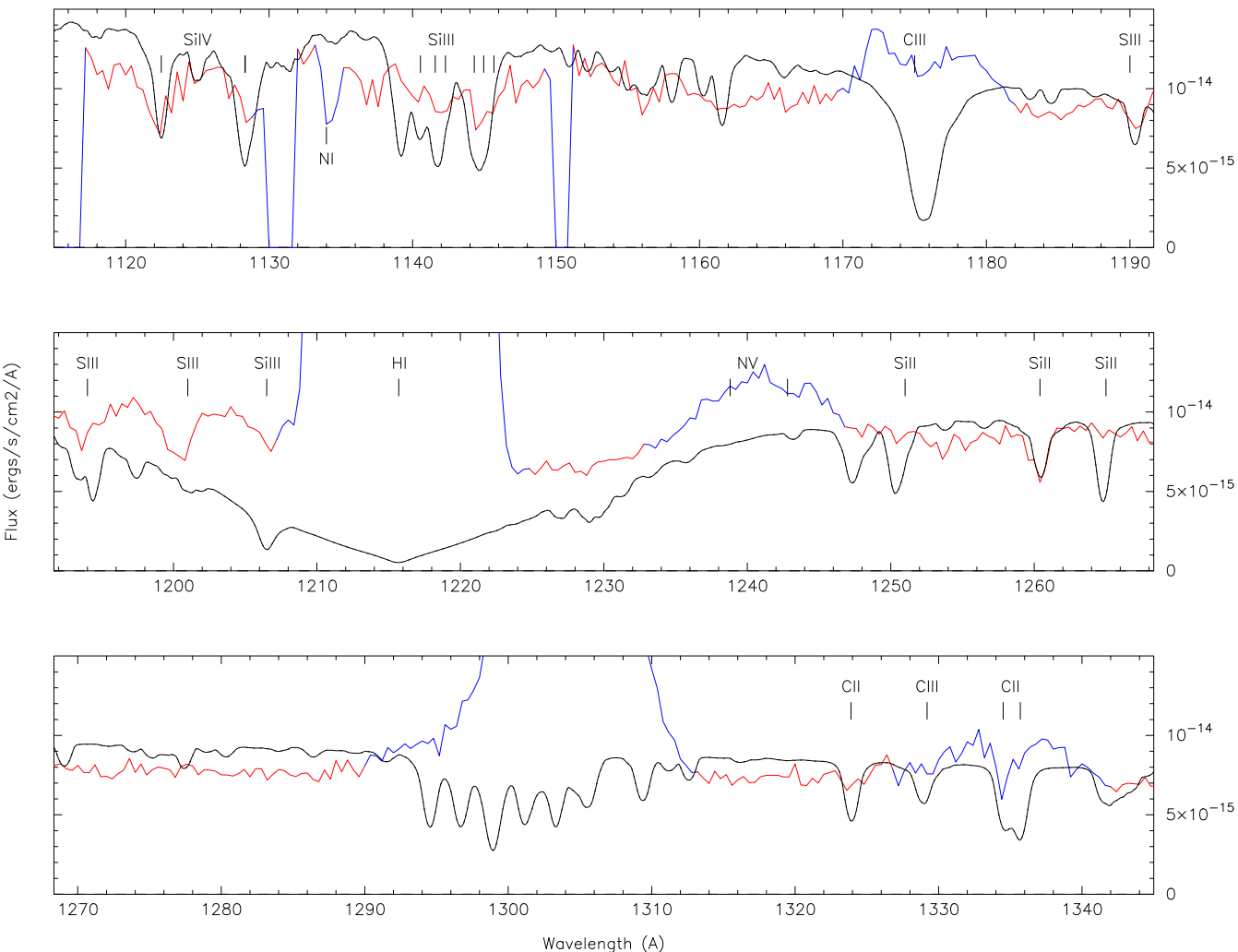

Figure 5. Same as in Figure 4, but with a stellar rotational velocity of $150 \mathrm{~km} \mathrm{~s}^{-1}$ to fit the Si II $1260 \AA$ line. The short-wavelength region is shown in detail with line identification. The Si II $1260 \AA$ line is the only line that can be matched with this WD model. The Si II 1251 and $1265 \AA$ lines are not observed. The C III (1175 $\mathrm{A})$ line is contaminated with broad emission, but its absorption feature on top of the emission is too shallow compared to the theoretical spectrum. These are clear indications that the observed lines do not form in the stellar WD photosphere.

account for the observed flux and will help us identify the lines forming in the WD photosphere (if any).

We first try to fit the slope of the continuum, and we find that it agrees well with a $23,000 \mathrm{~K}$ WD (our model \#1, see Table 1), but the Ly $\alpha$ region of the model forms a large broad absorption feature not seen in the observed spectrum. The distance obtained for this model is also far too short, only 291 pc, and $\chi_{\nu}^{2}=3.07$.

As we increase the temperature, we find a better agreement in the $\mathrm{Ly} \alpha$ region, but the model becomes too blue as its slope becomes steeper. The distance also increases, and it reaches $\sim 1 \mathrm{kpc}$ for a 50,000 K WD (model \#6), with $\chi_{\nu}^{2}=2.17$.

The model with least $\chi^{2}$ is obtained for a $30,000 \mathrm{~K} \mathrm{WD}$ (model \#3), with a distance of $515 \mathrm{pc}$. This model is shown in Figure 4. Even for this model the Ly $\alpha$ region is also too broad and too deep and the continuum is too steep. It is likely that the Ly $\alpha$ region $(1170-1250 \AA)$ is entirely dominated by broad emission lines. We note that setting the stellar rotational velocity to $150 \mathrm{~km} \mathrm{~s}^{-1}$ in this model gives a reasonable fit to the Si II $1260 \AA$ line. The short-wavelength region is shown in detail with line identification in Figure 5. However, the Si II $1260 \AA$ line is the only line that can be matched with this WD model. The expected Si II 1251 and $1265 \AA$ lines, as seen in the theoretical spectrum, are not observed. The C III (1175 $\AA$ ) line is contaminated with broad emission, but its absorption feature on top of the emission is too shallow compared to the theoretical spectrum.
We find that we can fit some set of lines by changing the WD temperature, but each temperature provides a fit to only a few lines. For example, in Figure 6, we set the WD temperature to $T=35,000 \mathrm{~K}$, with a projected rotational velocity of $300 \mathrm{~km} \mathrm{~s}^{-1}$, and obtain a relatively good fit to the Si IV (1122.5 and 1128.3 ̊) and Si III (1140.6, 1141.6, 1142.3, and $\sim 1145 \AA$ ) lines. However, the Si II (1260 $⿱$ ) line is not reproduced in this model.

Similarly, a WD temperature of $T=40,000 \mathrm{~K}$ with a projected rotational velocity of $150 \mathrm{~km} \mathrm{~s}^{-1}$ is needed to fit the C II 1324 and $\sim 1335 \AA$ absorption lines; however, none of the other lines are fitted.

These are clear indications that the observed lines do not form in the stellar WD photosphere. Overall, the single WD model does not provide a satisfactory fit. The results for the fits to the single WD model are recapitulated in the first part of Table 1 (models \#1-6).

\subsection{Single Disk Modeling}

As the absorption lines cannot satisfactorily be fitted with single WD models, we first check whether the disk alone can provide such a fit. We find that, in order to fit the narrow absorption lines in the COS spectrum, the inclination of the system would have to be extremely small $\left(\sim 3^{\circ}\right)$. That is, the disk would have to be almost face-on to minimize the broadening of the lines from the Keplerian velocity in the disk. Since there is no indication that this is the case, we deduce that the lines do not form in the disk either. The width of the 

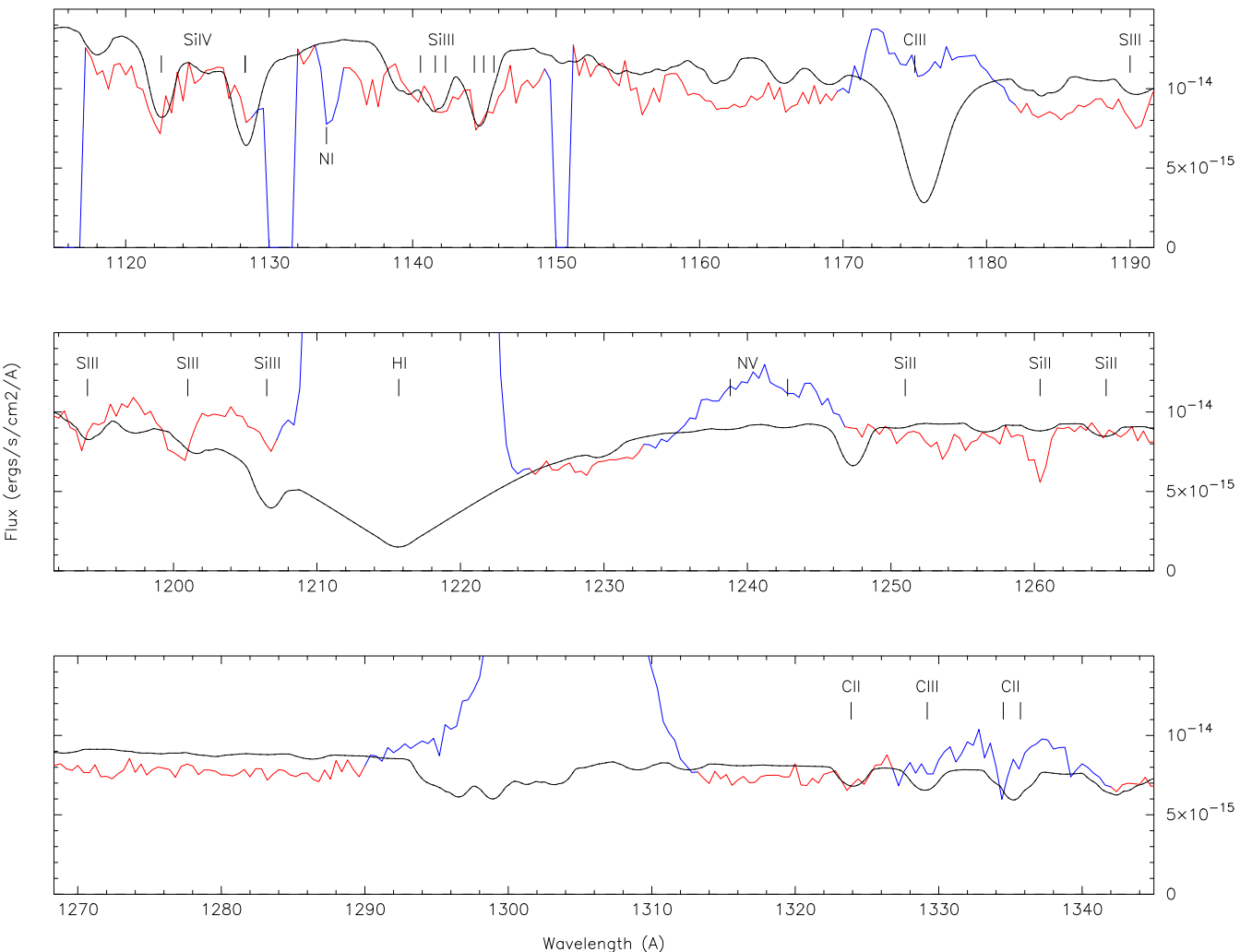

Figure 6. WD model fit to the Si IV and Si III absorption lines in the very short-wavelength region $(\lambda<1150 \AA)$ of the COS spectrum of BB Dor. The WD has a temperature $T=35,000 \mathrm{~K}$, gravity $\log (g)=8.4$, solar composition, and projected rotational velocity of $300 \mathrm{~km} \mathrm{~s}^{-1}$. The Si IV (1122.5 and $1128.3 \AA$ ) and Si III $(1140.6,1141.6,1142.3$, and $\sim 1145 \AA$ ) lines are relatively well fitted but the Si II (1260 $⿱$ ) line is not reproduced.

C IV emission line implies an inclination of $\sim 20^{\circ}$; we therefore set the inclination to $i=18^{\circ}$ (the smallest value in our grid of disk models). We vary the mass accretion rate from $\dot{M}=10^{-8.0} M_{\odot} \mathrm{yr}^{-1}$ down to $\dot{M}=10^{-10.5} M_{\odot} \mathrm{yr}^{-1}$ in logarithmic steps of 0.5 (models \#7-12 in Table 1, second part). Here, we make no further attempt to fit the absorption lines, but rather we concentrate on the continuum.

The best fit is for $\dot{M}=10^{-8.5} M_{\odot} \mathrm{yr}^{-1}$ (model \#8), with $\chi_{\nu}^{2}=1.32$, but it gives an unacceptably large distance (more than $4 \mathrm{kpc}$ ) and the spectral features at the shorter wavelengths are not reproduced by the disk model. This model is presented in Figure 7.

The slope of the observed spectrum agrees better with a disk model with $\dot{M}=10^{-9.5} M_{\odot} \mathrm{yr}^{-1}$ (\#10, Figure 8), which has a distance $(1356 \mathrm{pc})$ in better agreement with the $\approx 1-2 \mathrm{kpc}$ quoted above. However, the $\chi_{\nu}^{2}$ value (1.87) of this model is significantly larger than for the model with $\dot{M}=10^{-8.5} M_{\odot} \mathrm{yr}^{-1}$. In the vicinity of the Ly $\alpha$ region (around $1170-1250 \AA$ ) the fit is rather poor.

Overall, the single disk model provides only a marginal improvement over the single WD model. However, due to the limits on the distance to the system, the mass accretion rate in the single disk model is constrained to be in the range $10^{-10.5} M_{\odot} \mathrm{yr}^{-1}<\dot{M}<10^{-9} M_{\odot} \mathrm{yr}^{-1}$.

\subsection{Combined WD + Disk Modeling}

Since neither the single WD nor the single disk models fit the spectrum adequately, we next attempt models with both components. We run each single disk model in combination with a WD with a temperature varying from 20,000 to $60,000 \mathrm{~K}$, in steps of $1000 \mathrm{~K}$, a total of almost 200 model fits. We present here only the best-fit models.

Since the disk models with a large mass accretion rate gave an unacceptably large distance, we present here only the disk + WD models with lower mass accretion rate. For completeness we list some disk + WD models with large mass accretion rate in Table 1 (models \#13-18).

As the mass accretion rate is set to $\dot{M}=10^{-9.5} M_{\odot} \mathrm{yr}^{-1}$ the disk model is improved with the addition of a hot WD. In Figure 9 we present such a model with $T_{\mathrm{wd}}=35,000 \mathrm{~K}$, giving a distance of $1.5 \mathrm{kpc}$, and where the WD contributes $\approx 1 / 5$ of the FUV flux and the disk contributes the remaining $4 / 5$. The projected rotational velocity of the WD is $V_{\text {rot }} \sin i=200 \mathrm{~km} \mathrm{~s}^{-1}$. This model (\# 20 in Table 1) has $\chi^{2}=1.49$. As the WD temperature is further increased, to $T_{\mathrm{wd}}=48,000 \mathrm{~K}$ (model \#21) and above $(60,000 \mathrm{~K}$, model $\# 22$ ), the model provides a small improvement with $\chi_{\nu}^{2} \approx 1.3$.

The fit can be further improved to $\chi_{\nu}^{2} \approx 1.2$ by decreasing the mass accretion rate further: $\dot{M}=10^{-10} M_{\odot} \mathrm{yr}^{-1}$ with $T_{\text {wd }}$ $=40,000-50,000 \mathrm{~K}$. Models \#24-26 are all very similar and we present model \#24 in Figure 10. These models give a distance of the order of 1-1.2 kpc.

As we further decrease $\dot{M}$ to $\dot{M}=10^{-10.5} M_{\odot} \mathrm{yr}^{-1}$ $\left(\approx 3 \times 10^{-11} M_{\odot} \mathrm{yr}^{-1}\right)$, the lowest $\chi_{\nu}^{2}(1.23)$ for that mass accretion rate is obtained with $T_{\mathrm{wd}}=35,000 \mathrm{~K}$-model \#29. This model is presented in Figure 11 and gives a distance of $761 \mathrm{pc}$. However, this model has a WD contributing more than 
BB_Dor
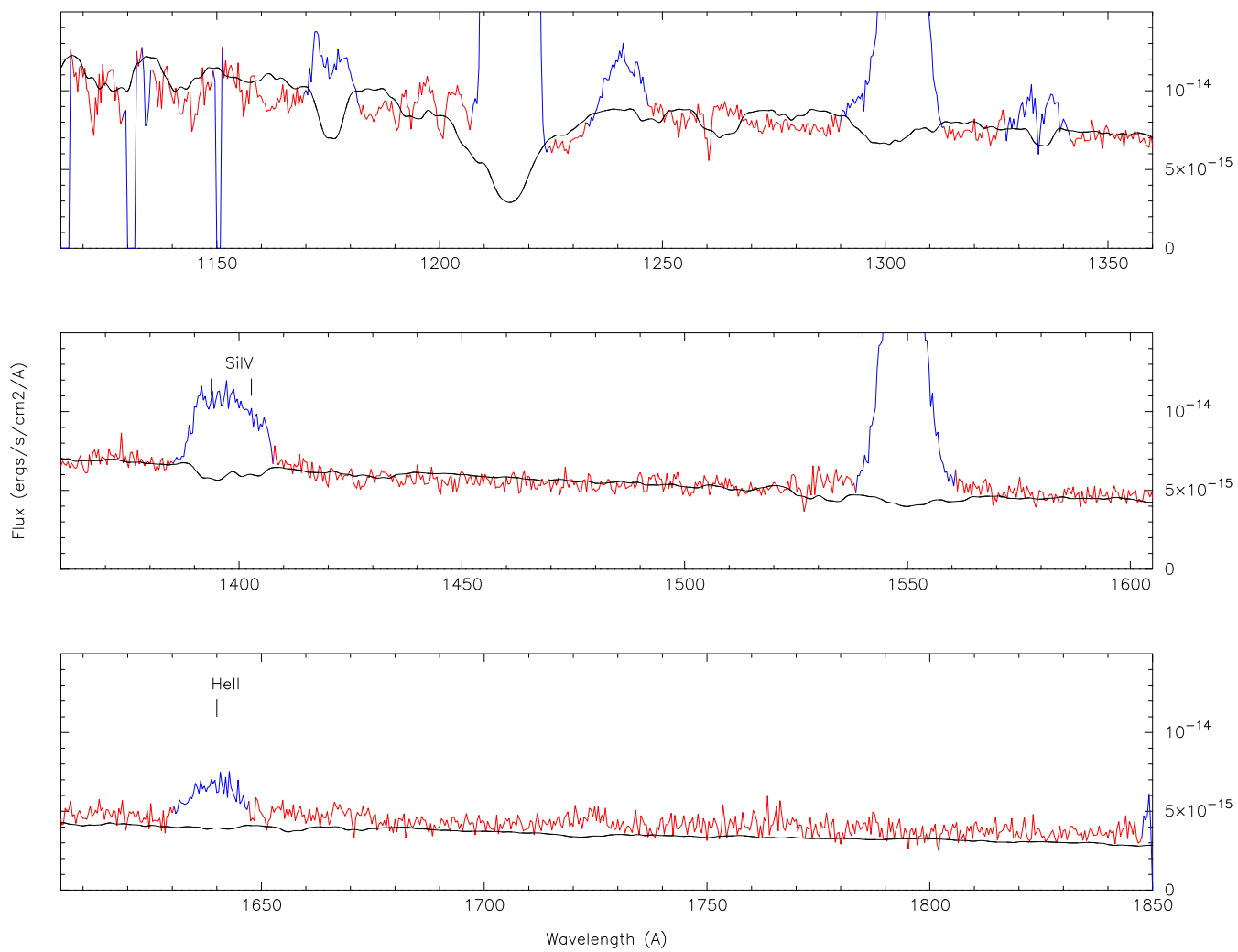

Figure 7. Single disk model fit to the COS spectrum of BB Dor, with $M_{\mathrm{wd}}=0.8 M_{\odot}, \dot{M}=10^{-8.5} M_{\odot} \mathrm{yr}^{-1}$, and $i=18^{\circ}$. This is the best fit of a single disk model, but it gives far too large a distance of $4288 \mathrm{pc}$.

BB_Dor
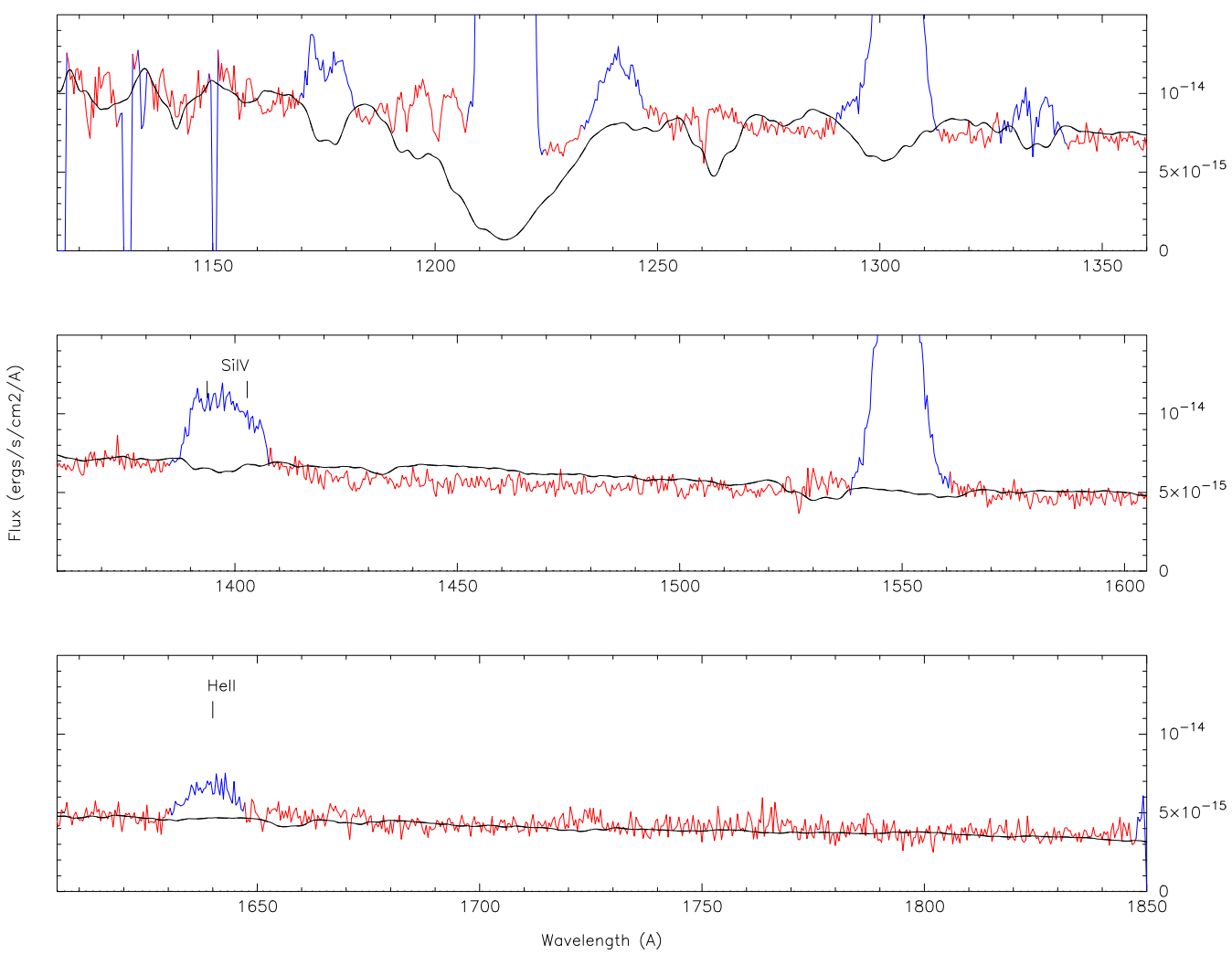

Figure 8. Single disk model fit to the COS spectrum of BB Dor, with $M_{\mathrm{wd}}=0.8 M_{\odot}, \dot{M}=10^{-9.5} M_{\odot} \mathrm{yr}^{-1}$, and $i=18^{\circ}$, giving a distance of 1356 pc. 

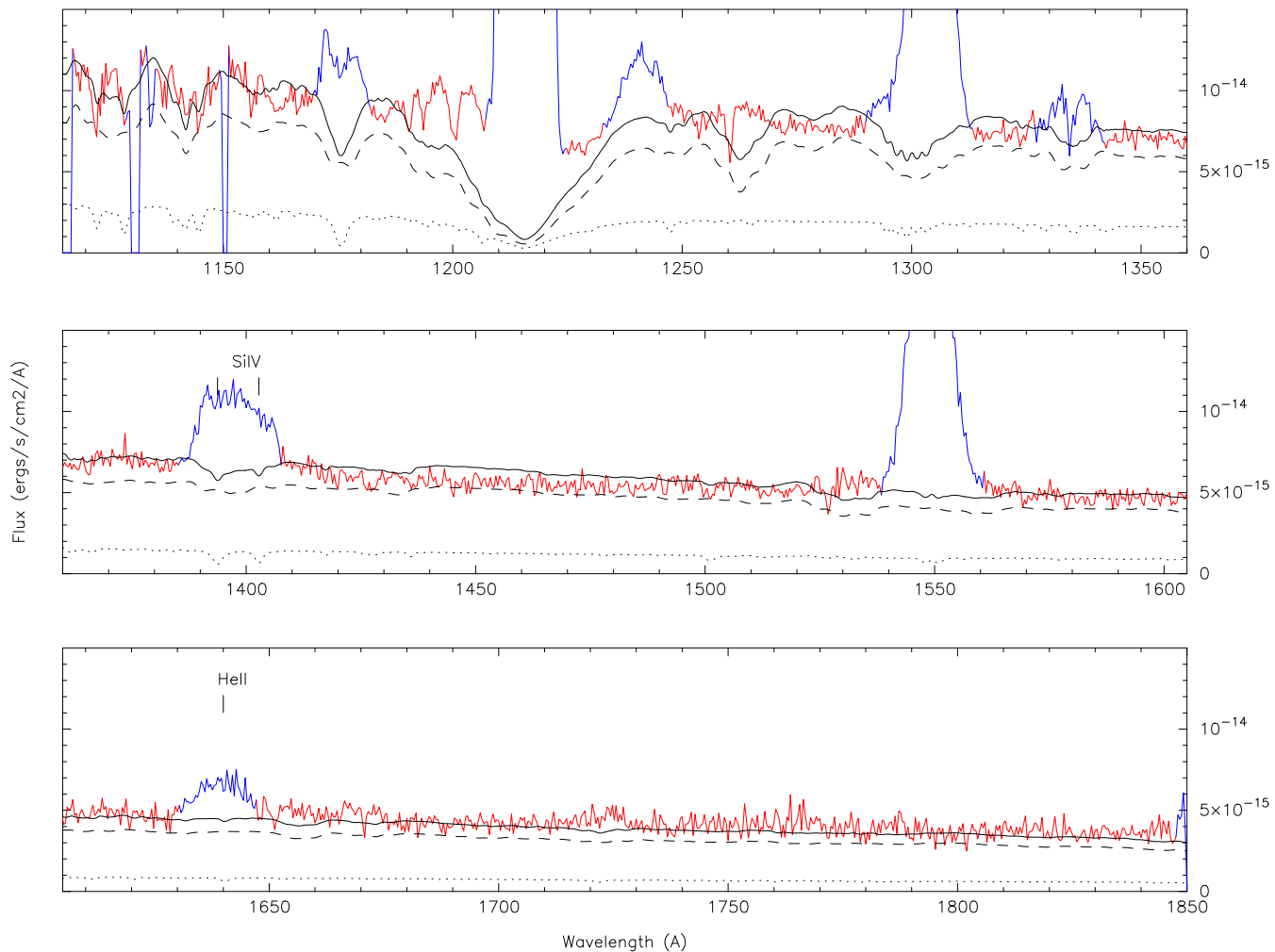

Figure 9. COS spectrum of BB Dor has been modeled (solid black line) with a disk with moderate mass accretion rate (dashed black line) plus a hot WD (dotted black line) to agree with the state in which it was observed. The WD contributes $21 \%$ of the FUV flux, and the disk contributes the remaining $79 \%$. Both the WD and the disk are assumed to have solar composition. The WD has a mass of $0.8 M_{\odot}$ and is accreting at a rate of $\dot{M}=10^{-9.5} M_{\odot} \mathrm{yr}^{-1} \approx 3 \times 10^{-10} M_{\odot} \mathrm{yr}^{-1}$, assuming an inclination of $i=18^{\circ}$. For this moderate mass accretion rate, a very good fit is obtained as long as the WD has a temperature of $T=35,000 \mathrm{~K}$ (shown here) or higher. The distance obtained from the fit is rather large, $d=1524 \mathrm{pc}$, and $\chi_{\nu}^{2}=1.49$. As the WD temperature is increased (up to $T \approx 60,000 \mathrm{~K}$ ), the fit is slightly improved $\left(\chi_{\nu}^{2} \approx 1.3\right)$, but the distance approaches its upper limit of $2 \mathrm{kpc}$.

$80 \%$ of the COS flux and therefore it has to be rejected. Other models with $\dot{M}=10^{-10.5} M_{\odot} \mathrm{yr}^{-1}$ have either a distance that is too short (when $T_{\mathrm{wd}}$ is decreased) or a WD contributing more than $80 \%$ of the flux (when $T_{\mathrm{wd}}$ is increased).

The best fits obtained for the combined WD + disk components have a mass accretion rate $\dot{M}=10^{-9.5}$ $10^{-10} M_{\odot} \mathrm{yr}^{-1}$ and WD temperature $T_{\mathrm{wd}} \sim 35,000 \mathrm{~K}$ or larger.

\section{DISCUSSION AND CONCLUSION}

We found that the absorption lines in the COS spectrum of BB Dor do not match the WD photosphere lines, and cannot originate in the disk. Absorption lines were also observed in the FUSE spectrum of BB Dor taken during its high state, when the disk dominated the FUV. Because of that the FUSE absorption lines could not originate in the WD photosphere, and, because of their width, they could originate in the disk only if $i<10^{\circ}$ (Godon et al. 2008). Since the inclination is probably larger, i.e., $\sim 20^{\circ}$, we conclude that the absorption lines observed in the FUV spectra of BB Dor during high and intermediate states must come either from material above the disk and WD, or from circumbinary material, or from the interstellar medium (ISM).

The ISM lines in the FUSE spectra of CVs are usually very narrow with a broadening $\ll 1 \AA$, consisting of low ionization species such as Fe II, S I, C II, Si II, and Ar I (Godon et al. 2006, 2012), while the absorption features in the COS spectrum of
BB Dor have a larger velocity broadening $>1 \AA$. Nonetheless, broader ISM lines have been observed in the FUV spectra of some CVs (Maucher et al. 1988), which enable us to tentatively identify the following lines as being interstellar in origin: Si II

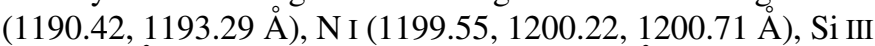
(1206.51 А), S II (1250.59, 1253.81, $1259.52 \AA)$, Si II (1260.42 $\AA)$, and C II (1334.53, $1335.70 \AA)$.

No hydrogen Ly $\alpha$ absorption profile was discernible due to the broad emission in the spectral region 1170-1250 $\AA$. Other regions of the spectrum also exhibit some broad and strong emission lines. As a consequence, and also due to the unknown mass and uncertain distance estimate, the modeling of the COS spectrum of BB Dor proved to be challenging.

In our modeling we assumed an inclination $i=18^{\circ}$, a standard CV WD mass of $0.8 M_{\odot}$, solar abundances, and an upper limit of $2 \mathrm{kpc}$ for the distance (Rodríguez-Gil et al. 2012). The combined WD plus accretion disk models with a disk with moderately low mass accretion rate and a hot WD appear to be the best fits as far as the lowest $\chi_{\nu}^{2}$ value is concerned.

These results have to be further considered while taking into account that the system was in an intermediate state with a flux 20 times lower than during the FUSE observation, when the system was in its high state (Godon et al. 2008). The FUSE spectrum of $\mathrm{BB}$ Dor had a continuum flux level decreasing below $1000 \AA$, implying that the mass accretion rate had to be $\approx 10^{-9} M_{\odot} \mathrm{yr}^{-1}$ at the time of the FUSE observation, because 

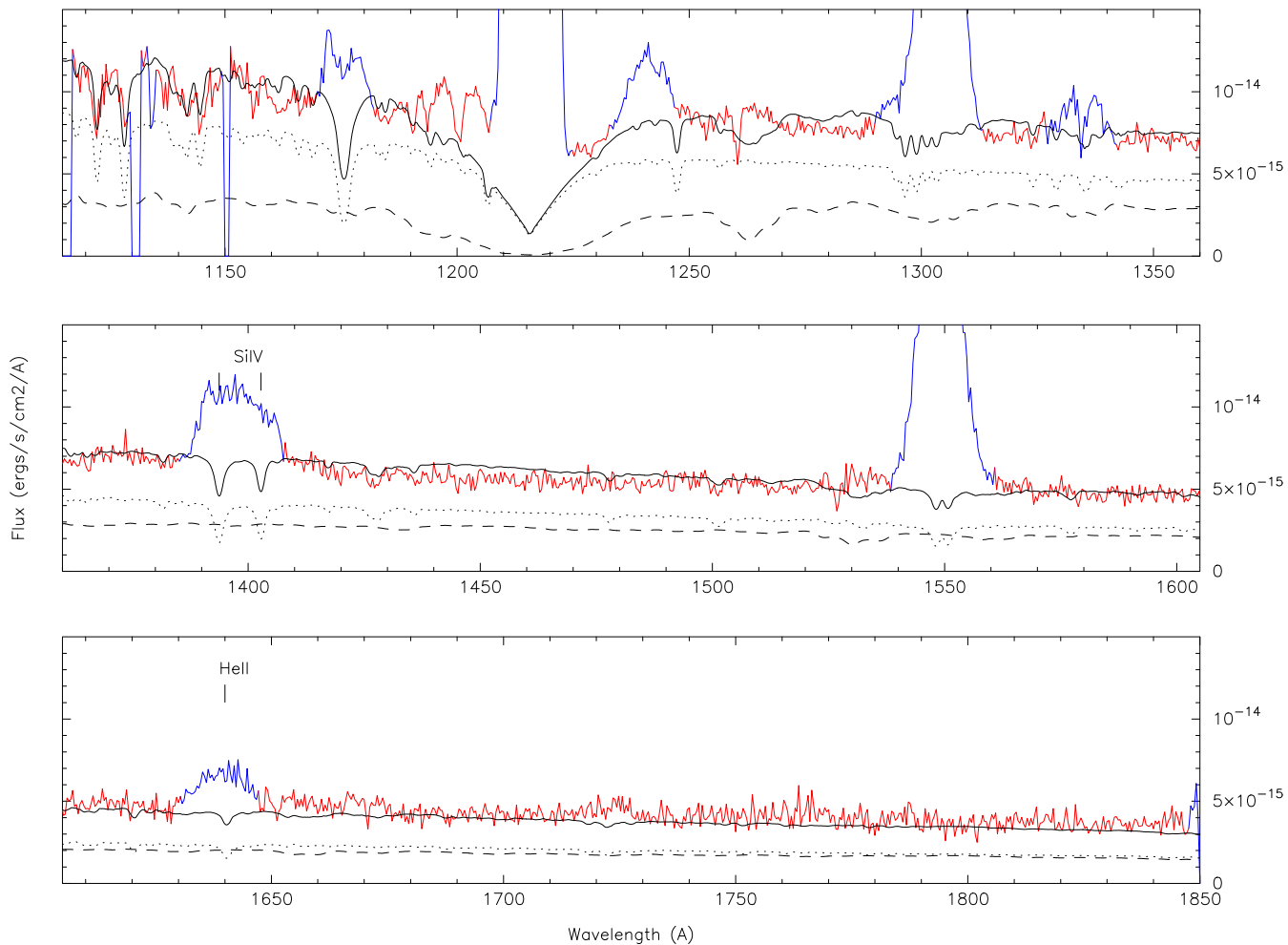

Figure 10. Combined WD plus disk model of the COS spectrum of BB Dor. The WD has a temperature $T=40,000 \mathrm{~K}$, a projected rotational velocity $V_{\text {rot }} \sin i=200 \mathrm{~km} \mathrm{~s}^{-1}$, and a mass of $0.8 M_{\odot}$. The mass accretion rate is $\dot{M}=1 \times 10^{-10} M_{\odot} \mathrm{yr}^{-1}$ with $i=18^{\circ}$. The distance obtained is $d=1094 \mathrm{pc}$, and the reduced $\chi_{\nu}^{2}=1.21$. The WD contributes $65 \%$ of the FUV flux, and the disk contributes the remaining $35 \%$. Here too, as long as the WD temperature is large (up to $T \sim 50,000 \mathrm{~K})$, the fit remains very good $\left(\chi_{\nu}^{2}=1.2\right)$, and the distance increases to $1.2 \mathrm{kpc}$.

models with larger mass accretion rate $\left(\dot{M}>10^{-9} M_{\odot} \mathrm{yr}^{-1}\right)$ were hotter and contributed more flux at the shorter wavelengths $(<1000 \AA)$ of FUSE. The FUSE spectral fit also gave a distance estimate of $\approx 700 \mathrm{pc}$. At the time of the COS observation, BB Dor must have had a lower mass accretion rate, namely $\dot{M} \leqslant 10^{-9.5} M_{\odot} \mathrm{yr}^{-1}$ (i.e., three times lower than during the FUSE observations), and more likely $\dot{M} \approx 10^{-10} M_{\odot} \mathrm{yr}^{-1}$ (i.e., 10 times lower than during the FUSE observations).

As the WD cannot contribute more than a maximum of $\sim 80 \%$ of the continuum, which would be achieved in the extreme case where the disk luminosity itself varies by $100 \%$, we reject models \#29 and \#30, and due to the distance constraint models \#27 and \#28 also have to be rejected. In other words, during the COS observations, the mass accretion rate must have been of the order of $\dot{M}=10^{-10} M_{\odot} \mathrm{yr}^{-1}$ or larger.

We therefore conclude that BB Dor, during its COS observation, most probably had a mass accretion rate $\dot{M} \sim 10^{-10} M_{\odot} \mathrm{yr}^{-1}$ and a WD temperature of $\sim 35,000$ to $\sim 50,000 \mathrm{~K}$, as presented in Figure 10. This is typical of VY Scl NL systems, and is higher than expected for CVs just above the period gap. We note, however, that the distance obtained from the fit changes dramatically in the solutions, while its estimate is poorly constrained (from $\sim 700 \mathrm{pc}$ to about $2 \mathrm{kpc}$ ). Anticipated Gaia data will soon provide distances to many CVs, and should help resolve the best solution for BB Dor and many other CVs. A short distance $(<1 \mathrm{kpc})$ would imply a low mass accretion rate $\left(<10^{-10} M_{\odot} \mathrm{yr}^{-1}\right)$ with a WD temperature of $\sim 35,000 \mathrm{~K}$, while a large distance $(\sim 2 \mathrm{kpc})$ would imply larger mass accretion rate $\left(>10^{-10} M_{\odot} \mathrm{yr}^{-1}\right)$ and WD temperature $(T \sim 50,000 \mathrm{~K})$.

Only a few VY Scl NL systems have been observed in an intermediate state, and the modeling of their FUV spectra has shown mixed results. AC Cnc seems to reveal a disk and WD, though the mass accretion rate and WD temperature depend strongly on the unknown distance (Bisol et al. 2012). BZ Cam has an FUV spectrum consistent with a 12,500 K blackbody (Prinja et al. 2000), but it is a unique peculiar object exhibiting an emission bow-shock nebula (Ellis et al. 1984). MV Lyr was one of the few VY Scl systems that were modeled in a low state (Hoard et al. 2004; Godon et al. 2012), but the spectrum of its intermediate state could not be modeled with a standard disk model and part of the disk had to be set to a low isothermal temperature (Linnell et al. 2005). However, these mixed results are characteristic of NL systems in high state as well. While some NLs have been successfully modeled with standard disk spectra (e.g., IX Vel, V3885 Sgr, V794 Aql, RZ Gru; Godon et al. 2007; Linnell et al. 2007, 2009; Bisol et al. 2012) others appear impossible to model with standard disk spectra (e.g., RW Sex, V751 Cyg, V380 Oph, UX UMa, QU Car; Linnell et al. 2008a, 2008b, 2010; Zellem et al. 2009). The results of the statistical study of Puebla et al. (2007) suggest that the temperature profile of the standard disk model be revised at least in the innermost parts of the disk in order to properly model some of the disk-dominated CV systems. With, so far, no existing alternative disk model to the standard one, the current results for BB Dor are at least consistent with typical 

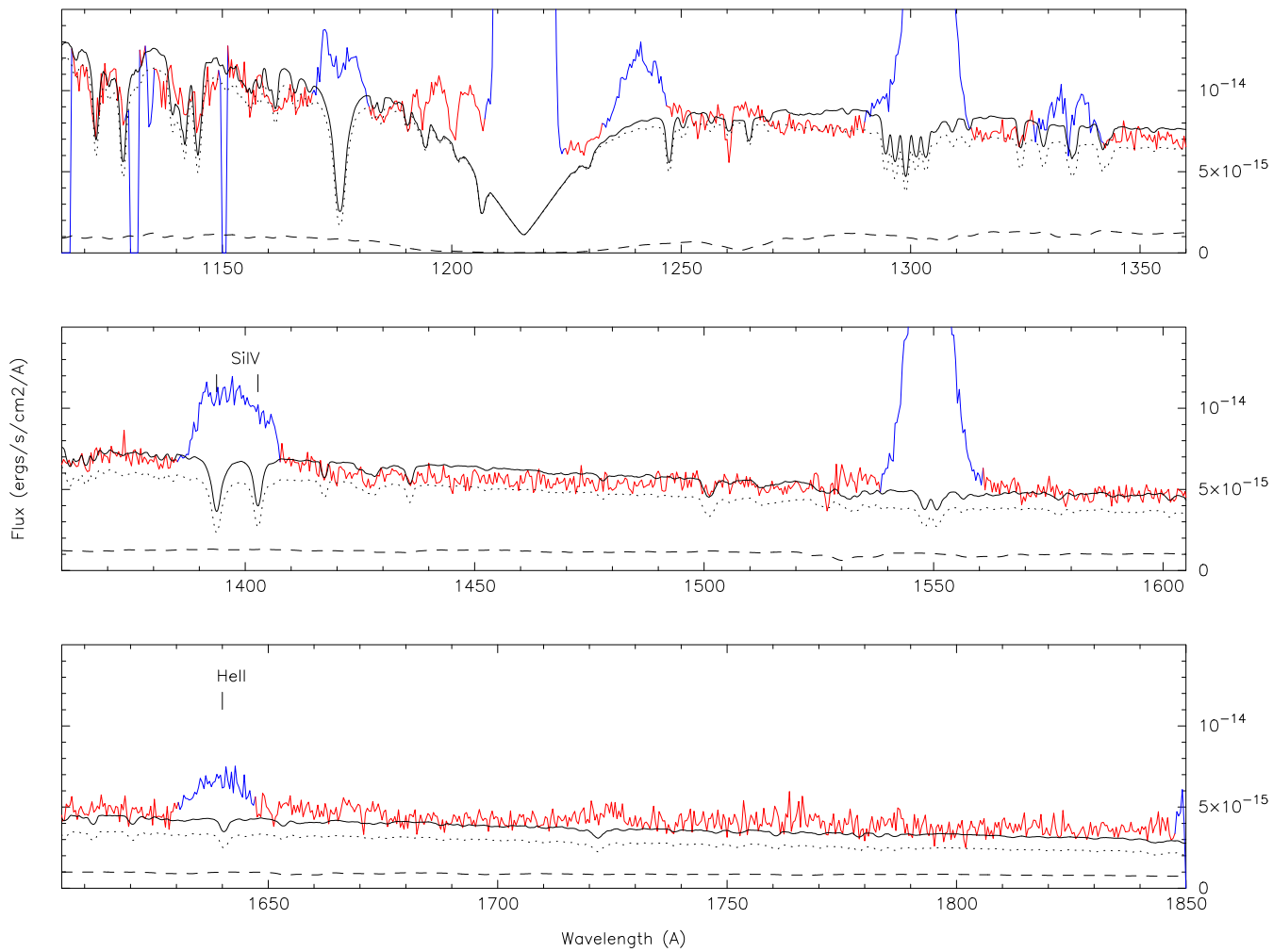

Figure 11. Combined WD plus disk model of the COS spectrum of BB Dor. The WD has a temperature $T=35,000 \mathrm{~K}$, a projected rotational velocity $V_{\text {rot }} \sin i=200 \mathrm{~km} \mathrm{~s}^{-1}$, and a mass of $0.8 M_{\odot}$. The disk has a mass accretion rate of $\dot{M}=3 \times 10^{-11} M_{\odot} \mathrm{yr}^{-1}$ and an inclination $i=18^{\circ}$. The distance obtained is $d=761 \mathrm{pc}$, and the reduced $\chi_{\nu}^{2}=1.23$. The WD contributes $86 \%$ of the FUV flux, and the disk contributes the remaining $14 \%$.

WD temperatures in VY Scl NLs, and improved disk models, as well as accurate distances obtained by Gaia in the near future, will provide better constraints on the physical parameters of this system.

We would like to thank Knox Long and Christian Knigge for reading and commenting on an early version of this manuscript. P.G. wishes to thank Bill Blair for his kind hospitality at the Rowland Department of Physics and Astronomy in the Johns Hopkins University, Baltimore, Maryland. Special thanks to the Austral Variable Star Observer Network (AVSON), and the American Association of Variable Star Observers (AAVSO) for their monitoring of BB Dor. This research is part of the GO program 12870 based on observations made with the NASA/ ESA Hubble Space Telescope, obtained at and funded by the Space Telescope Science Institute, which is operated by the Association of Universities for Research in Astronomy Inc., under NASA contract NAS 5-26555. The research leading to these results has received funding from the European Research Council under the European Union's Seventh Framework Programme (FP/2007-2013)/ERC Grant Agreement n.320964 (WDTracer). D.d.M. acknowledges financial support from ASI INAF contract I/037/12/0.

\section{REFERENCES}

Bisol, A. C., Godon, P., \& Sion, E. M. 2012, PASP, 124, 158

Chen, A., O’Donoghue, D., Stobie, R. S., Kilkenny, D., \& Warner, B. 2001, MNRAS, 325, 89

Ellis, G. L., Grayson, E. T., \& Bond, H. E. 1984, PASP, 96, 283

Godon, P., Sion, E. M., Barrett, P. E., \& Szkody, P. 2007, ApJ, 656, 1092
Godon, P., Sion, E. M., Barrett, P. E., Szkody, P., \& Schlegel, E. M. 2008, ApJ, 687, 532

Godon, P., Sion, E. M., Cheng, F., et al. 2006, ApJ, 642, 1018

Godon, P., Sion, E. M., Levay, K., et al. 2012, ApJS, 203, 29

Goliasch, J., \& Nelson, L. 2016, ApJ, 809, 80

Hack, M., \& la Dous, C. 1993, Cataclysmic Variables and Related Objects (Washington, DC: US Gov. Printing Office), 95

Hoard, D. W., Linnell, A. P., Szkody, P., et al. 2004, ApJ, 604, 346

Hodge, P. E. 2011, in ASP Conf. Ser. 442, Astronomical Data Analysis Software and Systems XX, ed. I. N. Evans et al. (San Fransisco, CA: ASP), 391

Hodge, P. E., Keyes, C., \& Kaiser, M. E. 2007, BAAS, 39, 972

Howell, S., Nelson, L., \& Rappaport, S. 2001, ApJ, 550, 897

Hubeny, I. 1988, CoPhC, 52, 103

Hubeny, I., \& Lanz, T. 1995, ApJ, 439, 875

Knigge, C., Baraffe, I., \& Patterson, J. 2011, ApJ, 194, 28

la Dous, C. 1990, in NASA/CNRS Monograph Series on Non-Thermal Phenomena in Stellar Atmospheres, ed. M. Hack, (Washington, DC: NASA; Paris: CNRS), 14

la Dous, C. 1991, A\&A, 252, 100

Linnell, A. P., Godon, P., Hubeny, I., et al. 2008a, ApJ, 676, 1226

Linnell, A. P., Godon, P., Hubeny, I., et al. 2009, ApJ, 703, 1839

Linnell, A. P., Godon, P., Hubeny, I., Sion, E. M., \& Szkody, P. 2007, ApJ, 662, 1204

Linnell, A. P., Godon, P., Hubeny, I., Sion, E. M., \& Szkody, P. 2008b, ApJ, 688,568

Linnell, A. P., Godon, P., Hubeny, I., Sion, E. M., \& Szkody, P. 2010, ApJ, 719,271

Linnell, A. P., Szkody, P., Gänsicke, B. T., et al. 2005, ApJ, 624, 923

Maucher, C. W., Raymond, J. C., \& Córdova, F. A. 1988, ApJ, 335, 829

Pala, A. F., Gänsicke, B. T., Townsley, D., et al. 2016, MNRAS, submitted

Patterson, J. 2002, Center for Backyard Astrophysics Communication, (http:// cbastro.org/communications/news/messages/0278.html)

Press, W. H., Teukolsky, S. A., Vetterling, W. T., \& Flannery, B. P. 1992, Numerical Recipes in Fortran 77, The Art of Scientific Computing (2nd ed; Cambridge: Cambridge Univ. Press)

Pringle, J. E. 1981, ARA\&A, 19, 137 
Prinja, R. K., Ringwald, F. A., Wade, R. A., \& Knigge, C. 2000, MNRAS, 312, 316

Puebla, R. E., Diaz, M. P., \& Hubeny, I. 2007, AJ, 134, 1923

Rappaport, S., Joss, P. C., \& Webbink, R. F. 1982, ApJ, 254, 616

Rappaport, S., Verbunt, F., \& Joss, P. C. 1983, ApJ, 275, 713

Rodríguez-Gil, P., Schmidtobreick, L., Long, K. S., et al. 2012, MNRAS, 422, 2332

Rodríguez-Gil, P., Shahbaz, T., Marsh, T. R., et al. 2015, MNRAS, 452, 146

Schmidtobreick, L., Rodríguez-Gil, P., Long, K. S., et al. 2012, MNRAS, 422,731
Shakura, N. I., \& Sunyaev, R. A. 1973, A\&A, 24, 337

Starrfield, S. 1971, MNRAS, 152, 307

Townsley, D. M., \& Bildsten, L. 2005, ApJ, 628, 395

Townsley, D. M., \& Gänsicke, B. T. 2009, ApJ, 693, 1007

Wade, R. A., \& Hubeny, I. 1998, ApJ, 509, 350

Warner, B. 1995, Cataclysmic Variable Stars (Cambridge: Cambridge Univ. Press)

Wood, M. A. 1990, PhD thesis, Univ. Texas

Zellem, R., Hollon, N., Ballouz, R.-L., et al. 2009, PASP, 121, 942

Zorotovic, M., Schreiber, M. R., \& Gänsicke, B. T. 2011, A\&A, 536, 42 